\title{
Emergency department impaired adherence to personal protective equipment donning and doffing protocols during the COVID-19 pandemic
}

Tomer Lamhoot ${ }^{1}$, Noa Ben Shoshan', Hagit Eisenberg', Gilad Fainberg ${ }^{1}$, Mansour Mhiliya ${ }^{1}$, Neta Cohen²,3, Orly Bisker-Kassif ${ }^{1}$, Orly Barak ${ }^{4}$, Carolyn Weiniger ${ }^{5}$ and Tali Capua ${ }^{3^{*}}$ (D)

\begin{abstract}
Objectives: Coronavirus Disease 2019 (COVID-19) is a highly infectious viral pandemic that has claimed the lives of millions. Personal protective equipment (PPE) may reduce the risk of transmission for health care workers (HCWs), especially in the emergency setting. This study aimed to compare the adherence to PPE donning and doffing protocols in the Emergency Department (ED) vs designated COVID-19 wards and score adherence according to the steps in our protocol.

Design: Prior to managing COVID-19 patients, mandatory PPE training was undertaken for all HCWs. HCWs were observed donning or doffing COVID-19 restricted areas.

Setting: Donning and doffing was observed in COVID-19 designated Emergency department and compared to COVID-19 positive wards.

Participants: All HCWs working in the aforementioned wards during the time of observation.

Results: We observed 107 donning and doffing procedures (30 were observed in the ED). 50\% HCWs observed donned PPE correctly and 37\% doffed correctly. The ED had a significantly lower mean donning score (ED: 78\%, Internal: 95\% ICU: 96\%, $p<0.001$ ); and a significantly lower mean doffing score (ED: 72\%, Internal: 85\% ICU: 91\%, $p=0.02$ ).

Conclusions: As hypothesized, HCWs assigned to the designated ED wing made more protocol deviations compared with HCWs positive COVID-19 wards. Time management, acuity, lack of personnel, stress and known COVID-19 status may explain the lesser adherence to donning and doffing protocols. Further studies to assess the correlation between protocol deviations in use of PPE and morbidity as well as improvement implementations are required. Resources should be invested to ensure PPE is properly used.
\end{abstract}

Keywords: Personal protective equipment, COVID-19, Emergency department

\footnotetext{
* Correspondence: talicapua@gmail.com

${ }^{3}$ Pediatric Emergency Medicine, Dana-Dwek Children's Hospital, Tel Aviv, Israel, affiliated to the Sackler Faculty of Medicine, Tel Aviv University, Tel Aviv, Israel

Full list of author information is available at the end of the article
}

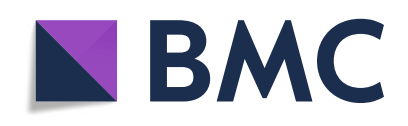

(- The Author(s). 2021 Open Access This article is licensed under a Creative Commons Attribution 4.0 International License, which permits use, sharing, adaptation, distribution and reproduction in any medium or format, as long as you give appropriate credit to the original author(s) and the source, provide a link to the Creative Commons licence, and indicate if changes were made. The images or other third party material in this article are included in the article's Creative Commons licence, unless indicated otherwise in a credit line to the material. If material is not included in the article's Creative Commons licence and your intended use is not permitted by statutory regulation or exceeds the permitted use, you will need to obtain permission directly from the copyright holder. To view a copy of this licence, visit http://creativecommons.org/licenses/by/4.0/. The Creative Commons Public Domain Dedication waiver (http://creativecommons.org/publicdomain/zero/1.0/) applies to the data made available in this article, unless otherwise stated in a credit line to the data. 


\section{Introduction}

Personal protective equipment (PPE) is defined as barrier clothing, gloves, eye protection and/or headgear designed to protect from a potential hazardous exposure. In medicine, these exposures are typically either infectious diseases or toxins (1). The transmission of the Severe acute respiratory syndrome coronavirus 2 (SARS-CoV-2), and its disease, COVID-19, is presumed to be primarily through droplets and fomites, although viral particles have also been found in feces of seropositive patients. A recent study by Van Doremalen et al. suggested aerosol and fomite as a mode of SARS-CoV-2 transmission, and reinforced the reported high spread rate (2). In hospital settings, performing aerosolgenerating procedures (e.g. intubation, suction, bronchoscopy and cardiopulmonary resuscitation) facilitated patientto-healthcare worker $(\mathrm{HCW})$ transmission $(3,4)$.

The COVID-19 pandemic has highlighted the need for appropriate PPE to reduce transmission risks for HCWs caring for COVID-19 patients (5). Reports from Italy's experience dealing with COVID-19 suggest that up to $20 \%$ of healthcare professionals dealing with COVID-19 patients became infected with the virus, with some reported deaths (6). China's experience also found $41 \%$ of patients in one hospital acquired COVID-19 in hospital (7). Furthermore, Adams et al. reported that 3000 HCWs were infected with COVID-19 and 22 died (8).

International guidelines exist for $\mathrm{HCW}$ s regarding PPE use to minimize COVID-19 transmission $(9,10)$, and precautions during donning and doffing are recommended in all guidelines (4).

PPE donning and doffing entails specific steps, reportedly taking 7 to $15 \mathrm{~min}$ for donning and 14 to 23 for doffing (11).

A review of the literature between 2014 to 2020 reported $\mathrm{HCW}$ protocol deviations both during donning and doffing when using PPE, with consequential contamination risks $(5,12-15)$.

To the best of our knowledge, in-situ PPE donning and doffing has not been studied in designated COVID19 wards. Our objective was to assess adherence of PPE donning and doffing protocols, and compare Emergency Department (ED) assessments to non-emergency departments. Due to the suspected status of the patients, high volume of patients with variable degrees of acuity and inappropriate staff to patient ratios, we hypothesized that protocol deviations would be more likely to occur in COVID-19 ED wing and less likely in COVID-19 confirmed floors (i.e. Intensive Care Unit - ICU and internal medicine ward).

\section{Methods}

\section{Study design}

This study comprised three groups; teams entering and exiting the COVID-19 suspected ED wing, COVID-19 confirmed ICU and COVID-19 internal medicine ward.

\section{Study protocol}

A checklist was created, based on the hospital protocol for donning and doffing PPE (Appendices 1a and 1b). This protocol is based on the guidelines of the World Health Organization (WHO) and the Centers for Disease Control and Prevention (CDC) regarding PPE for COVID-19 $(9,10)$. Donning was assessed in eleven steps, and doffing in eight steps with an extra step for those wearing eyeglasses. Mandatory PPE donning and doffing training was undertaken for all HCWs between March 4 and April 10, 2020. Prior to managing COVID-19 suspected/positive patients, all HCWs participated in a group, frontal demonstration and practice. A short video clip was distributed to all hospital staff. A poster (Additional file 1) was placed in all departments, and the poster and video were available via the hospital COVID-19 mobile app.

Observers were trained by the epidemiology department to teach and observe donning and doffing. Observations were conducted between April 20th and April 26th, 2020. Time slots for observations were chosen randomly but included all hours of the day, all days of the week. The HCWs were not alerted to observations taking place to reduce possible procedural bias however, all wards had PPE adherence staff in place during the time of the observations.

A sample donning and doffing event was calculated by all members of the team to assess intra-team validity. Donning and doffing was scored according to the correct performance of each item thus the maximum score for donning was 11 and for doffing was 8 (9 if wearing eyeglasses). We used a detailed checklist to minimize intraobserver variation.

Our Institutional Review Board waived the need for approval and consent due to the nature of the project.

\section{Study setting, population and sample size}

This study was performed in a-1100 bed, tertiary care center between March 4 and April 26, 2020 as part of a quality improvement project during the COVID-19 pandemic.

Observations of $107 \mathrm{HCWs}$ were performed in COVID-19 designated departments - internal medicine ward (Internal; $62 \mathrm{HCWs}$ ) and intensive care unit (ICU) for confirmed positive COVID-19 cases (15 HCWs) and a designated emergency department (ED) wing for suspected or confirmed COVID-19 cases (30 HCWs). All three departments had designated areas for both donning and doffing. Observations were recorded according to the checklist (Appendices 1a and 1b).

Inclusion criteria: HCWs assigned to COVID-19 designated wards and ED, entering or exiting the restricted area when observers were present. 


\section{Data analysis}

All checklist records were collected via Microsoft Excel 2016 (version 16.0.4266.1001). Mean donning and doffing score were our primary outcome. Generalized Linear Models (GLM) were calculated and analyzed with R software (version 3.6.1) to predict PPE donning and doffing mean score based on department type, worker type and their interaction.

\section{Results}

One hundred and seven HCW observations were made; 56 during donning and 51 during doffing out of which, 19 and 11 HCWs in the ED respectively. HCWs included: nurses $(n=50)$, attending physicians $(n=4)$, resident doctors $(n=$ $11)$, intern doctors $(n=7)$, physiotherapists $(n=3)$, janitors $(\mathrm{n}=4)$, patient transport staff $(n=16), X-$ Ray technicians $(n=7)$, paramedics $(n=1)$, and administrative staff $(n=2)$, in three different departments: COVID-19 positive internal medicine department (Internal; $n=62$ ), suspected COVID19 designated Emergency Department (ED; $n=30$ ), and the COVID-19 positive Intensive care unit (ICU; $n=15$ ).

Additional file 1 presents the PPE steps for donning. $50 \%$ donned their PPE without protocol deviations (16\% entering the ED, 57\% HCWs entering the ICU and 70\% entering the internal medicine ward). Major omissions included failure to remove ID badges, jewelry and cell phones; failure to disinfect hands in at least one of the required steps, and neglecting to don gloves over cuffs. Figure $1 \mathrm{~A}$ refers to donning by step. During donning, $41 \% \mathrm{HCWs}$ failed to use hand sanitizer in at least one step. $32 \%$ of HCWs in the ED, failed to don gloves properly.

We used a Generalized Linear Model (GLM) with quasibinomial distribution and a logit link function to predict donning mean score based on department type and worker type. We found that the emergency department had a significantly lower mean donning score (ED: 78\%, Internal: 95\% ICU: 96\%, $\beta=-1.2, \mathrm{SE}=0.29, p<0.001$ ). Figure $2 \mathrm{~A}$ shows the donning score by department. No differences were found between worker types.

Overall, 37\% doffed their PPEs appropriately. Figure $1 \mathrm{~B}$ refers to doffing by step. Gaps were observed in all steps; HCWs neglected to remove gloves, visor, cap, gown and N95 mask properly and failed to disinfect hands in at least one of the 3 steps. While doffing, 12\% of all HCWs observed failed to disinfect their hands in at least one of 3 of the steps (steps 2, 6 and 8). Most cases of failure to disinfect hands in one of the 3 steps

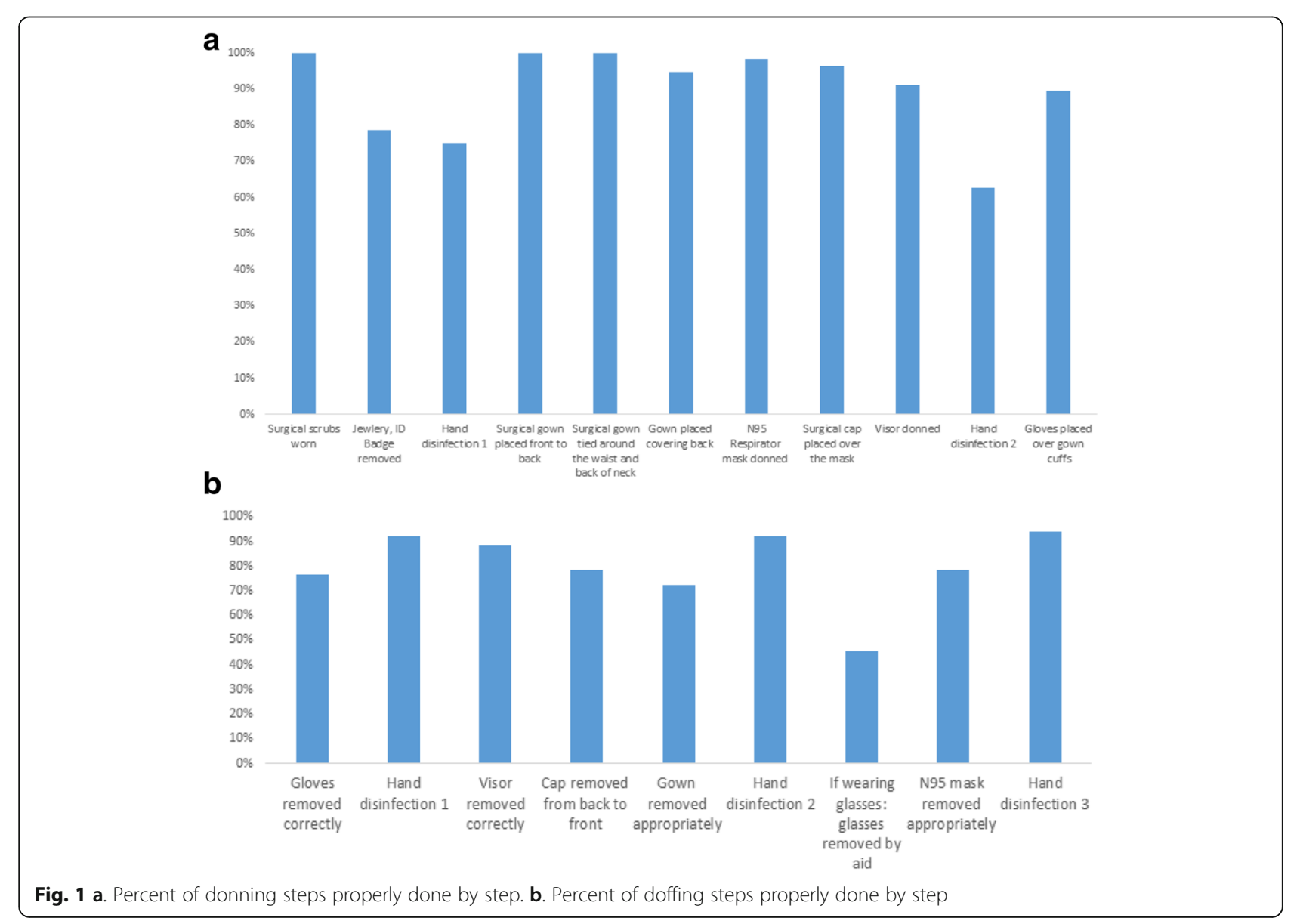




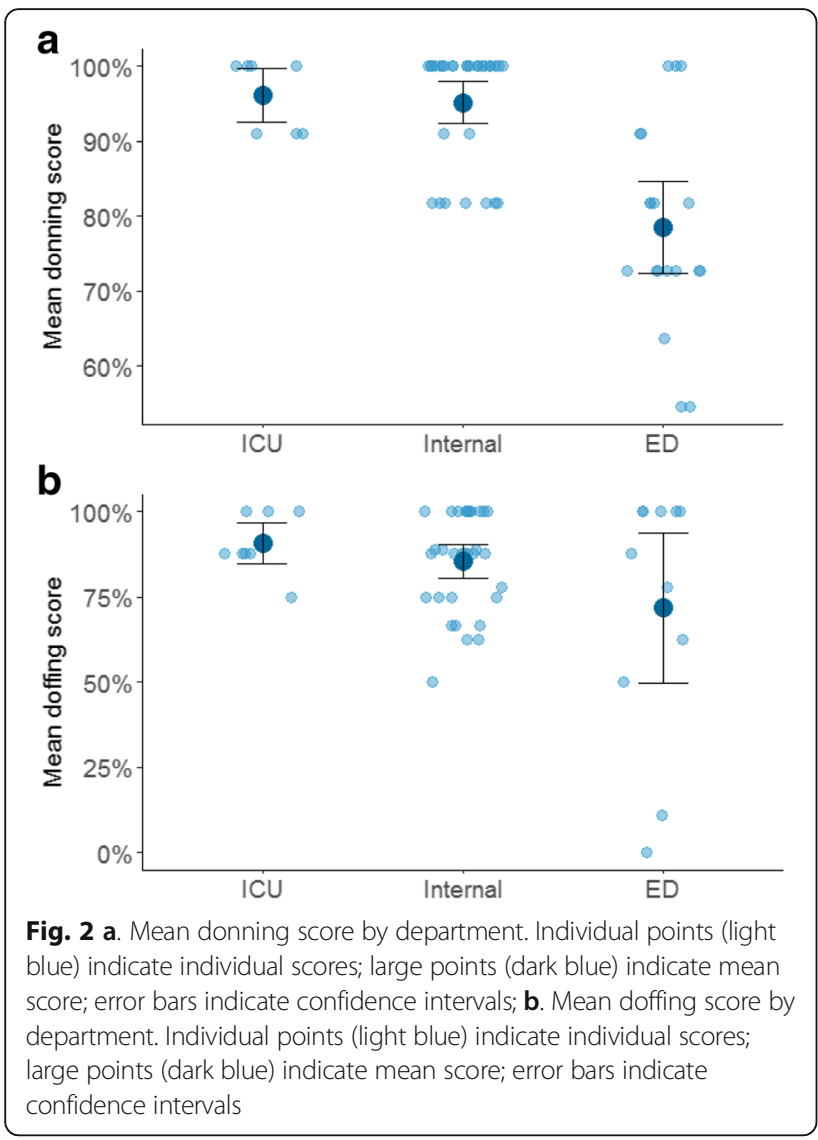

took place in the ED (73\%). Of the $11 \mathrm{HCWs}$ wearing glasses, 5 (45\%) appropriately doffed by having someone else remove the glasses before removing the N-95 mask. $78 \%$ of all observed HCWs removed the N95 respirator mask appropriately.

We used a Generalized Linear Model (GLM) with quasibinomial distribution and a logit link function to predict donning mean score based on department type and worker type. The emergency department had a significantly lower mean doffing score (ED: $72 \%$, Internal: $85 \%$ ICU: $91 \%, \beta=-0.82$, $\mathrm{SE}=0.35, p=0.02$ ). Figure $2 \mathrm{~B}$ shows the doffing score in the different departments. No differences were found between the worker types.

\section{Discussion}

In our observational study of donning and doffing steps in COVID-19 departments, we report the overall rate for fully correct donning and doffing to be $44 \%$.

This concerning finding is similar to prior studies observing PPE use $(5,11,16,17)$. Kwon at al reported that protocol deviations were common in both donning and doffing, and found that 100\% of Ebola Virus Disease HCWs committed at least one PPE protocol deviation during doffing and $27 \%$ while donning (5). Casalino et al. reported that PPE doffing protocol deviations occurred even after a three-phase training program (11).

During the SARS-CoV-2 pandemic, and specifically within the time period in which our study took place, strategies to improve adherence had not yet been implemented. Failure to improve the success rate may have a profound effect on HCWs morbidity. Protocol deviations in donning and doffing may have multifactorial causes. Gaps between the development of Infection Protection and Control guidelines, their introduction to the target audience, and their implementation $(4,18)$, likely occur for a number of reasons. Firstly, HCWs may lack sufficient practice and simulation time $(11,18)$; a review by Houghton et al. (2020) have suggested support from managers, workplace culture, physical space, access to and trust in PPE, and a desire to deliver good patient care as additional factors to affect guidelines following (18). Secondly, high consequence infectious diseases have been shown to produce anxiety among HCWs which may hinder performance (19). Moreover, it is both intuitive and evidence based that performing medical tasks that require psychomotor skill would be more difficult when wearing PPE (1). Previous studies have assessed the impact of PPE on resuscitative efforts. Chen et al. found that wearing PPE in a resuscitation scenario significantly deteriorates the quality of chest compression and may thus deteriorate outcome and survival (20). Castle et al. concluded in their study that intubation and intravenous cannulation attempts are adversely affected by wearing PPE (21).

We found that the ED had significantly lower mean donning and doffing scores, potentially attributable to the suspected status of the patients, as opposed to the designated wards with COVID-19 confirmed positive patients. Moreover, fatigue from frequent PPE changes, high volume of patients with variable degrees of acuity and inappropriate staff-to-patient ratio combined with the negative impact of PPE on resuscitative efforts, as discussed before, may have a great influence on ED HCWs and explain our findings.

Our study highlights PPE donning and doffing errors. We believe there is a need for intensive practice and regular observations for appropriate PPE use, especially for ED teams. In the past, simulation and training methods were studied and significantly improved the trainee's proficiency (11). For example, Abualenain et al. reported in their PPE simulation-based training, that the average score using checklist upon encounter suspected case of Ebola improved significantly after simulation training (22). Although beyond the scope of this current paper, yet of higher importance, is the work that has and should be done in order to better the adherence of HCWs to PPE protocols. 


\section{Limitations}

This study had several limitations. Although we assume that pre-observation performance rates were much lower, compared to the post observation rates, we did not compare pre observation and post observation rates. Moreover, our hospital used only one style of long gown PPE and protocol (see additional file 1) during the time our study took place. Other types of PPE or different protocols may influence the error rate. A recent review published in the Cochrane database summarizes the differences between overalls, long gowns and aprons and their overall donning and doffing quality (23). Varbeek et al. found that although covering more of the body was shown to protect the HCW during care for affected patients, these PPEs were associated with less comfort, and increased difficulty in donning and doffing offering more opportunities for transmission.

\section{Conclusions}

PPE is a vital element used not only to combat pandemics but also in the daily dealing with common pathogens requiring contact precaution. Resources need to be extensively invested to assure implementation of PPE donning and doffing protocols in order to improve HCWs and patients' protection. PPE type needs to be selected based on ease of care, comfort and training capabilities. Finally, a special focus needs to be invested in ED teams due to the special circumstances they confront with: high volume of patients with variable levels of acuity and unknown COVID-19 status.

Authors' contributions The author(s) read and approved the final manuscript.

\section{Supplementary Information}

The online version contains supplementary material available at https://doi. org/10.1186/s13584-021-00477-7

\section{Additional file 1.}

\section{Funding}

This research received no specific grant from any funding agency, commercial or not-for-profit sectors.

\section{Competing interests}

None.

\section{Author details}

${ }^{1}$ Medical Technology and Simulation Center, Tel Aviv Sourasky Medical Center, affiliated to Ministry of Health, Tel Aviv, Israel. ${ }^{2}$ Pediatric Emergency Medicine, The hospital for Sick Children, Toronto, Canada. ${ }^{3}$ Pediatric Emergency Medicine, Dana-Dwek Children's Hospital, Tel Aviv, Israel, affiliated to the Sackler Faculty of Medicine, Tel Aviv University, Tel Aviv, Israel. ${ }^{4}$ Department of Geriatrics, Tel Aviv Sourasky Medical Center, Tel Aviv, Israel affiliated to the Sackler Faculty of Medicine, Tel Aviv University, Tel Aviv, Israel. ${ }^{5}$ Department of Anesthesia, Tel Aviv Sourasky Medical Center, Tel Aviv, Israel, affiliated to the Sackler Faculty of Medicine, Tel Aviv University, Tel Aviv, Israel.
Received: 16 November 2020 Accepted: 21 April 2021

Published online: 19 July 2021

\section{References}

1. Adler MD, Krug S, Eiger C, Good GL, Kou M, Nash M, et al. Impact of Personal Protective Equipment on the Performance of Emergency Pediatric Tasks. Pediatr Emerg Care [Internet]. 2020 Feb 24. Volume Publish Ahead of Print - Issue Available from: https://doi.org/10.1097/PEC.0000000000002028.

2. van Doremalen $\mathrm{N}$, Bushmaker T, Morris DH, Holbrook MG, Gamble A, Williamson BN, et al. Aerosol and surface stability of SARS-CoV-2 as compared with SARS-CoV-1. N Engl J Med [Internet]. 2020;382(16):1564-7. Available from:. https://doi.org/10.1056/NEJMc2004973.

3. Varia M, Wilson S, Sarwal S, McGeer A, Gournis E, Galanis E, et al. Investigation of a nosocomial outbreak of severe acute respiratory syndrome (SARS) in Toronto, Canada. CMAJ [Internet]. 2003;169(4):285-92 Available from: https://pubmed.ncbi.nlm.nih.gov/12925421/.

4. Islam eMS, Rahman KM, Sun Y, Qureshi MO, Abdi I, Chughtai AA, et al. Current knowledge of COVID-19 and infection prevention and control strategies in healthcare settings: A global analysis. Infect Control Hosp Epidemiol [Internet]. 2020; 41(10):1-11. Available from: https:/www.cambridge.org/core/product/identifier/ S0899823X20002378/type/journal_article

5. Kwon JH, Burnham C-AD, Reske KA, Liang SY, Hink T, Wallace MA, et al. Assessment of healthcare worker protocol deviations and selfcontamination during personal protective equipment donning and doffing. Infect Control Hosp Epidemiol [Internet]. 2017;38(9):1077-83. Available from: https://www.cambridge.org/core/product/identifier/S0899823X17001210/ type/journal_article. https://doi.org/10.1017/ice.2017.121.

6. Remuzzi A, Remuzzi G. COVD-19 and Italy: what next? Lancet [Internet]. 2020 Apr; 395(10231):1225-8. Available from: https:/linkinghub.elsevier.com/retrieve/pii/S014 0673620306279. https://doi.org/10.1016/S0140-6736(20)30627-9.

7. Wang D, Hu B, Hu C, Zhu F, Liu X, Zhang J, et al. Clinical Characteristics of 138 Hospitalized Patients With 2019 Novel Coronavirus-Infected Pneumonia in Wuhan, China. JAMA [Internet]. 2020;323(11):1061 Available from: https:// jamanetwork.com/journals/jama/fullarticle/2761044.

8. Adams JG, Walls RM. Supporting the Health Care Workforce During the COVID-19 Global Epidemic. JAMA [Internet]. 2020;323(15):1439 Available from: https://jamanetwork.com/journals/jama/fullarticle/2763136.

9. World Health Organization (WHO). Rational use of personal protective equipment for coronavirus disease 2019 (COVID-19) and considerations during severe shortages [Internet]. Who. 2020. p. 1-28. Available from: https://apps.who.int/iris/handle/10665/331695

10. Centers for Disease Control and Prevention (CDC). Interim Infection Prevention and Control Recommendations for Patients with Suspected or Confirmed Coronavirus Disease 2019 (COVID-19) in healthcare settings [Internet]. CDC. 2, 2020:1-10 Available from: https://www.cdc.gov/corona virus/2019-ncov/infection-control/control-recommendations.html.

11. Casalino E, Astocondor E, Sanchez JC, Díaz-Santana DE, del Aguila C, Carrillo JP. Personal protective equipment for the Ebola virus disease: a comparison of 2 training programs. Am J Infect Control [Internet]. 2015;43(12):1281-7. Available from: https://linkinghub.elsevier.com/retrieve/pii/S019665531 5007580. https://doi.org/10.1016/j.ajic.2015.07.007.

12. Verbeek JH, Rajamaki B, ljaz S, Sauni R, Toomeye E, Blackwood B, et al. Personal protective equipment for preventing highly infectious diseases due to exposure to contaminated body fluids in healthcare staff. Verbeek $\mathrm{JH}$, editor. Cochrane Database Syst Rev [Internet]. 2020; Available from: https:// doi.org/10.1002/14651858.CD011621

13. Kilmarx PH, Clarke KR, Dietz PM, Hamel MJ, Husain F, McFadden JD, et al. Ebola virus disease in health care workers--Sierra Leone, 2014. MMWR Morb Mortal Wkly Rep [Internet]. 2014;63(49):1168-71 Available from: http://www. ncbi.nlm.nih.gov/pubmed/25503921.

14. Lim SM, Cha WC, Chae MK, Jo IJ. Contamination during doffing of personal protective equipment by healthcare providers. Clin Exp Emerg Med [Internet] 2015;2(3):162-167. Available from: 10.15441/ceem.15.019

15. Matanock A, Arwady MA, Ayscue P, Forrester JD, Gaddis B, Hunter JC, et al. Ebola virus disease cases among health care workers not working in Ebola treatment units--Liberia, June-August, 2014. MMWR Morb Mortal Wkly Rep [Internet]. 2014;63(46):1077-81 Available from: http://www.ncbi.nlm.nih.gov/ pubmed/25412067.

16. Beam EL, Gibbs SG, Boulter KC, Beckerdite ME, Smith PW. A method for evaluating health care workers' personal protective equipment technique. Am J Infect Control [Internet]. 2011;39(5):415-20. Available from: https:// 
linkinghub.elsevier.com/retrieve/pii/S019665531000893X. https://doi.org/10.1 016/j.ajic.2010.07.009.

17. Zamora JE. Contamination: a comparison of 2 personal protective systems. Can Med Assoc J [Internet]. 2006;175(3):249-54. Available from:. https://doi. org/10.1503/cmaj.060094.

18. Houghton C, Meskell P, Delaney H, Smalle M, Glenton C, Booth A, et al. Barriers and facilitators to healthcare workers' adherence with infection prevention and control (IPC) guidelines for respiratory infectious diseases: a rapid qualitative evidence synthesis. Cochrane Database Syst Rev [Internet]. 2020 Apr 21; Available from: https://doi.org/10.1002/14651858.CD013582

19. White MK, Vercruyssen M, Hodous TK. Work tolerance and subjective responses to wearing protective clothing and respirators during physical work. Ergonomics [Internet]. 1989;32(9):1111-23. Available from:. https://doi. org/10.1080/00140138908966878.

20. Chen J, Lu K-Z, Yi B, Chen Y. Chest Compression With Personal Protective Equipment During Cardiopulmonary Resuscitation. Medicine (Baltimore) [Internet]. 2016;95(14):e3262 Available from: http://content.wkhealth.com/ linkback/openurl?sid=WKPTLP:landingpage\&an=00005792-201604050-00054.

21. Castle N, Owen R, Hann M, Clark S, Reeves D, Gurney I. Impact of chemical, biological, radiation, and nuclear personal protective equipment on the performance of low- and high-dexterity airway and vascular access skills. Theatr Res Int. 2009:80(11):1290-5 Available from: https://linkinghub.elsevier. com/retrieve/pii/S0300957209004158.

22. Abualenain JT, Al-Alawi MM. Simulation-based training in Ebola personal protective equipment for healthcare workers: experience from king Abdulaziz University hospital in Saudi Arabia. J Infect Public Health [Internet]. 2018;11(6): 796-800. Available from: https//linkinghub.elsevier.com/retrieve/pii/S1 876034118300704. https://doi.org/10.1016/j.jiph.2018.05.002.

23. Verbeek JH, Rajamaki B, ljaz S, Sauni R, Toomey E, Blackwood B, Tikka C, Ruotsalainen JH, Kilinc Balci FS. Personal protective equipment for preventing highly infectious diseases due to exposure to contaminated body fluids in healthcare staff. Cochrane Database Syst Rev 2020, Issue 4. Art. No.: CD011621. DOI: https://doi.org/10.1002/14651858.CD011621.pub4. .

\section{Publisher's Note}

Springer Nature remains neutral with regard to jurisdictional claims in published maps and institutional affiliations.

Ready to submit your research? Choose BMC and benefit from:

- fast, convenient online submission

- thorough peer review by experienced researchers in your field

- rapid publication on acceptance

- support for research data, including large and complex data types

- gold Open Access which fosters wider collaboration and increased citations

- maximum visibility for your research: over $100 \mathrm{M}$ website views per year

At $\mathrm{BMC}$, research is always in progress.

Learn more biomedcentral.com/submissions 